\title{
1040
}

The Vibroelectric Method - A New Tool for Nearsurface Characterisation and Improved Seismic Data Quality

\author{
T. Dean* (WesternGeco) \& J.C. Dupuis (Curtin University)
}

An understanding of the near surface is essential to improve the quality of seismic data by means of improved statics and near-surface models for migration. Recently attention has turned to using nonseismic methods to provide information about the near surface, but these methods require separate data acquisition programs and the integration of disparate physical measurements. The seismoelectric method promises to enable the acquisition of a near-surface model that can be directly integrated with seismic data without requiring a separate acquisition program. Most seismoelectric data were acquired using lowenergy impulsive sources that are inconsistent with commercial seismic surveys. This paper details the results of a test that shows that a vibratory source is suitable for seismoelectric surveys (the 'vibroelectric' method). We believe that the vibroelectric method has the potential to become a useful tool in both understanding the near surface and improving seismic data quality at very little additional cost. 


\section{Vienna |'11}

\section{Introduction}

The generation of electromagnetic fields by the passage of seismic waves through porous media is called the seismoelectric effect. The field is a result of the displacement of charged fluid contained in the pores of the rock. One source of electromagnetic arrivals is the coseismic field, a field generated by, and which travels coincident with, the seismic wave as it propagates. The coseismic field is consistent with the seismic field recorded by geophones and is thus of little interest. A second field of greater interest is called the interfacial response, and occurs when a P-wave encounters an interface between units of differing electric or mechanical properties. The P-wave causes the charge separation to be disturbed and results in what has been approximated to an oscillating electric dipole (Haartsen and Pride, 1997). This, in turn, generates a small electromagnetic disturbance.

An understanding of the near-surface is essential to improve the quality of seismic data by means of improved statics and near-surface models for migration. Recently, attention has turned to using nonseismic methods to provide information about the near-surface (e.g., Colombo and Keho, 2010). Such methods have two drawbacks:

1. They require a completely separate data acquisition program.

2. They require the integration of disparate physical measurements.

The seismoelectric method avoids these drawbacks because the data can be acquired concurrently with a standard seismic survey by simply adding additional sensors to the spread. Analysis of these seismoelectric measurements can be directly integrated with that of the seismic data.

The difficulty with application of the seismoelectric method is a generally poor signal-to-noise ratio (SNR). Nearly all the seismoelectric data to date have been acquired using impulsive sources with limited energy, typically a sledgehammer. The limited energy coupled with the weak interfacial response require the stacking of around 100 records to obtain an adequate SNR, even at typical target depths of less than $20 \mathrm{~m}$ (Haines, 2004). The most common source employed in commercial seismic surveys for oil exploration is the hydraulic vibrator (Sallas, 2010). The energy output of a hydraulic vibrator is orders of magnitude greater than that of a sledgehammer, suggesting that their use can increase the depth of investigation for seismoelectric surveys and/or reduce the number of records required to achieve the required SNR. This paper reports on a innovative recent experiment conducted to determine whether vibratory sources are suitable for seismoelectric surveys (or vibroelectric as we refer to the technique), i.e., that they are capable of generating seismoelectric interfacical signals.

\section{Data Acquisition}

Data were acquired between 4 and 6 November 2009, near Yanchep approximately $60 \mathrm{~km}$ north of Perth, Western Australia. The site was located on the Gnangara Mound, one of the most important groundwater resources for the Perth region.

The recording spread incorporated both electrical sensors and conventional geophones. The electrical sensors consisted of 40 dipoles, each made up of pairs of $50-\mathrm{cm}$ stainless steel stakes hammered into the ground with a 4-m spacing (Figure 1a). Each dipole was connected to a custom built differential preamplifier the size of a small lunch box. Data were initially acquired with dipole spacings of $4 \mathrm{~m}$ although this was later increased to $8 \mathrm{~m}$ (Figure 1b) to increase the size of the spread. When the electrode spacing was $4 \mathrm{~m}$ electrodes could be shared between adjacent dipoles (Figure 1a). The two sensor types were positioned on opposite sides of the 5-m wide road with a single geophone aligned with the western electrode. A Seistronix EX-6 24-bit distributed acquisition system was used to record the data. Cabling for the two receiver types was kept separated on either side of the road to eliminate the possibility of crosstalk. Interference between the two sensor types was not expected since previous experiments showed no cross-contamination between signals even when geophones were placed at the centre of the dipoles (Haines, 2004, p. 30). Data were recorded with a sample interval of $1 \mathrm{~ms}$ and an instrument gain of $24 \mathrm{~dB}$. 


\section{tor Vienna '11}

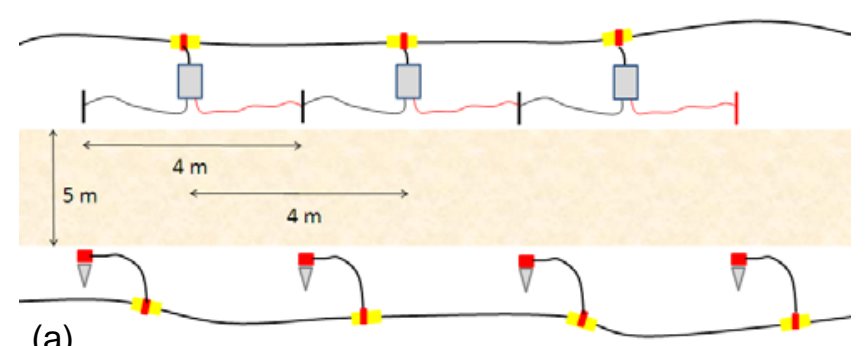

(a)

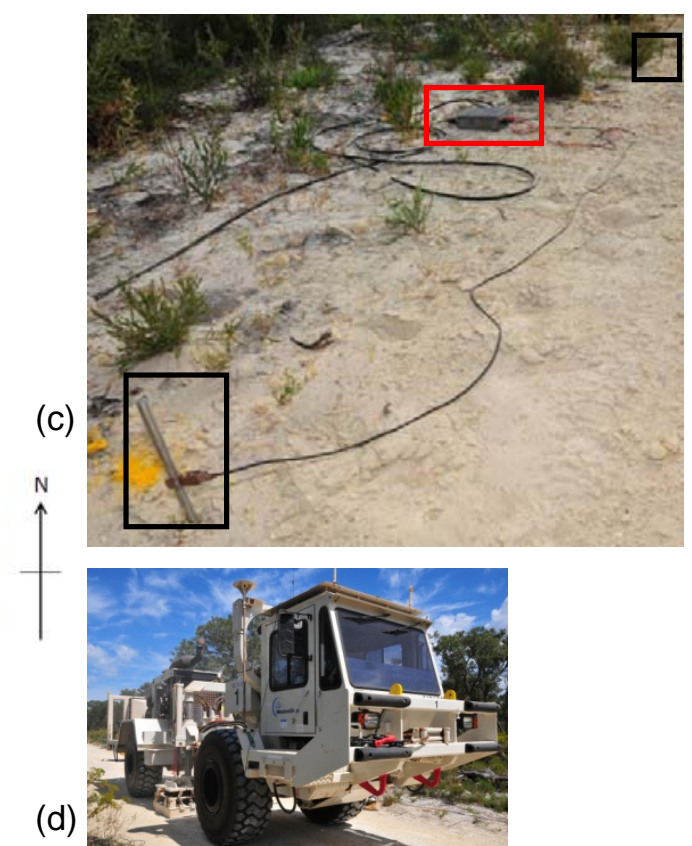

(b)

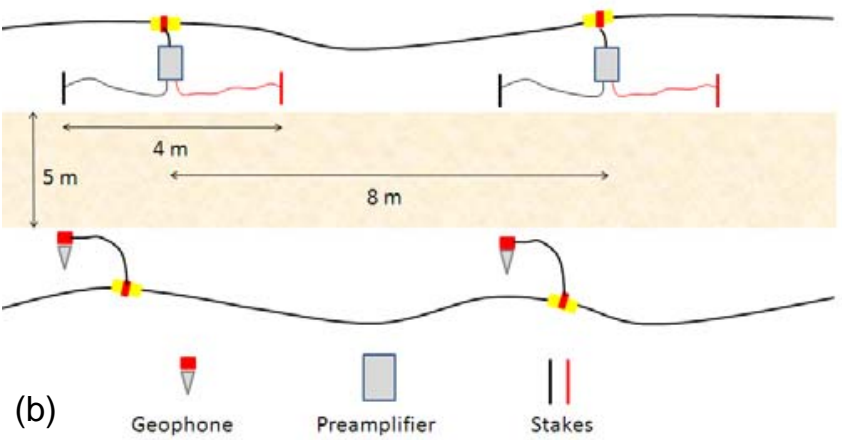

(c)

(d)

Figure 1 Illustration of a section of the vibroelectric and seismic recording geometry. The red box in c) highlights the preamplifier and the black boxes the electrodes (50-cm stainless steel stakes).

An AHV-IV 60,000-lb-peak-force vibrator unit was used as the source (Figure 1d). The vibrator pilot sweep was recorded by the acquisition system via a direct cable connection. The vibrator sweep used was linear 8 to $100 \mathrm{~Hz}$ over $12 \mathrm{~s}$ with 0.5 -s cosine tapers.

Several tests were conducted during the three days of acquisition. To increase the range of offsets available for analysis we acquired two walkaway tests where the source was initially positioned at the edge of the spread with subsequent source positions at 4-m intervals moving away from the spread (Figure 2a). Using the spread with 4-m dipole spacing we acquired data with a maximum offset of $258 \mathrm{~m}$, and with the 8-m dipole spacing we acquired data with a maximum offset of $436 \mathrm{~m}$ (Figure $2 \mathrm{~b})$. Using the 8-m dipole separation spread we acquired a 2D line. Source spacing was $4 \mathrm{~m}$ and we acquired a total of 192 records. We rolled the spread by six channels $(48 \mathrm{~m})$ nine times during the test giving a total line length of $768 \mathrm{~m}$. The data for the 2D line were also extended by incorporating the two walkaway tests. We also acquired 24 records at a source spacing of $1 \mathrm{~m}$ at the centre of the spread to allow the creation of composite shot or supergathers (Kepic and Rosid, 2004).

(a)
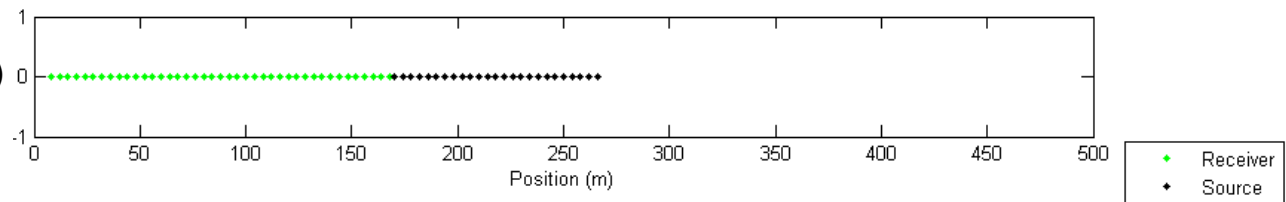

(b)

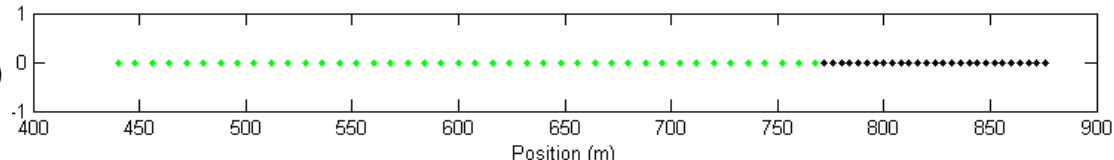

Figure 2 Walkaway test geometries for (a) the 4-m dipole-spaced spread and (b) the 8-m dipolespaced spread.

\section{Data Processing}

As found in other surveys power-line noise, typically concentrated at $50 \mathrm{~Hz}$, was significant but was successfully attenuated using a harmonic subtraction algorithm (Butler and Russell, 1993). No other data processing was performed on the data other than standard vibroseis correlation. 


\section{Vienna|'11}

\section{Results and Discussion}

Data from eight separate records acquired with 1-m source spacing were combined to create a 336trace supergather. Figure 3 shows both vibroelectric and seismic data supergathers created from the same records (the data were acquired simultaneously). The vibroelectric data exhibit many of the same events as the seismic data (a result of the coseismic field) but unlike the seismic data the events feature reversed polarity either side of the source as the field reaches the positive or negative electrode first.

As well as the coseismic field the vibroelectric data exhibits several events at times less than $100 \mathrm{~ms}$. Figure 4 shows the top $100 \mathrm{~ms}$ of both records for offsets between 60 and $100 \mathrm{~m}$ sorted by offset. Two flat events are clearly evident at 32 and 47 ms (indicated by the black arrows) that are not evident on the seismic data. These events have the characteristics of interfacial signals; they are effectively flat (they are generated directly beneath the source and propagate at the EM wave velocity so are received by the sensors almost simultaneously), and are polarity reversed either side of the source. Because the supergathers are the combination of eight different records the events cannot be the result of electrical noise because it would appear temporally random. A previous seismoelectric study in this area (Dupuis et al., 2007) found a similar response that was interpreted to be generated at the water table from a depth of approximately $14 \mathrm{~m}$.

Fast refractors, possibly associated with the base of the compressed road surface, obscured much of the data in the near traces and made deeper signals hard to identify. One possible signal at $80 \mathrm{~ms}$ is indicated by the orange arrow on Figure 3, but it is difficult to confirm.
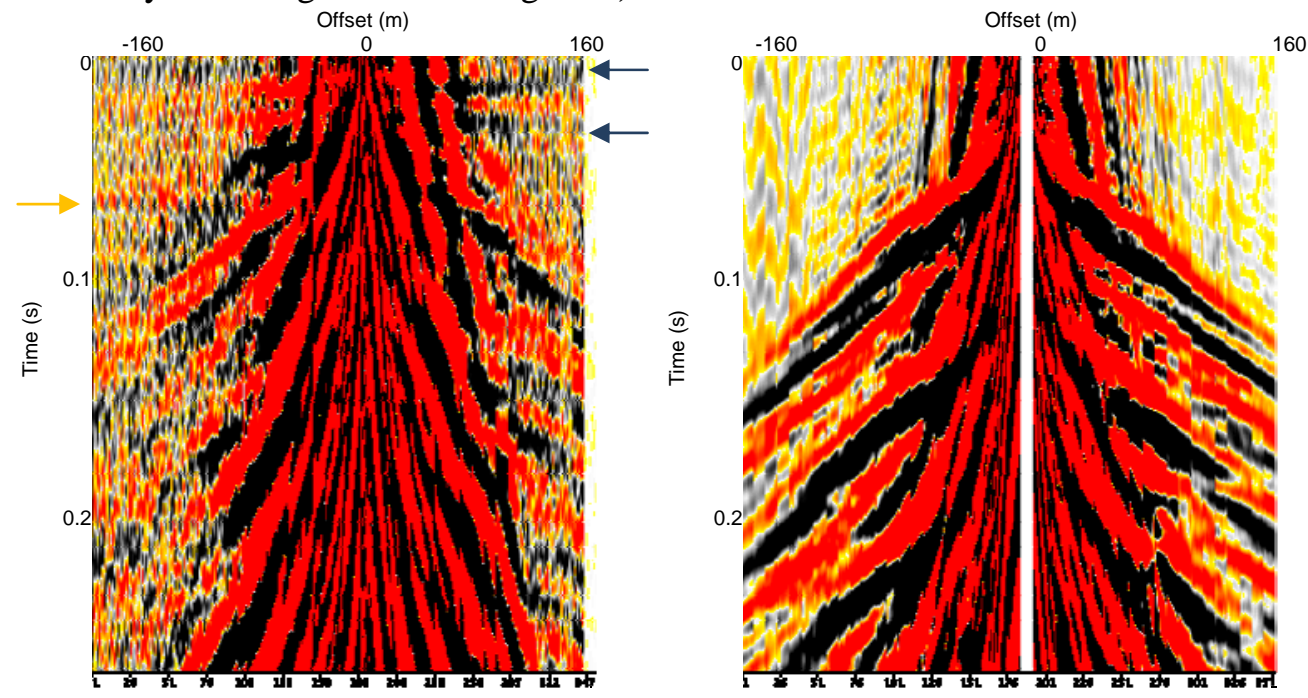

Figure 3 Vibroelectric (left) and seismic (rignt) supergathers created by combining eight records. The trace spacing is $1 \mathrm{~m}$. The scale of the seismic data is 50 times that of the vibroelectric.

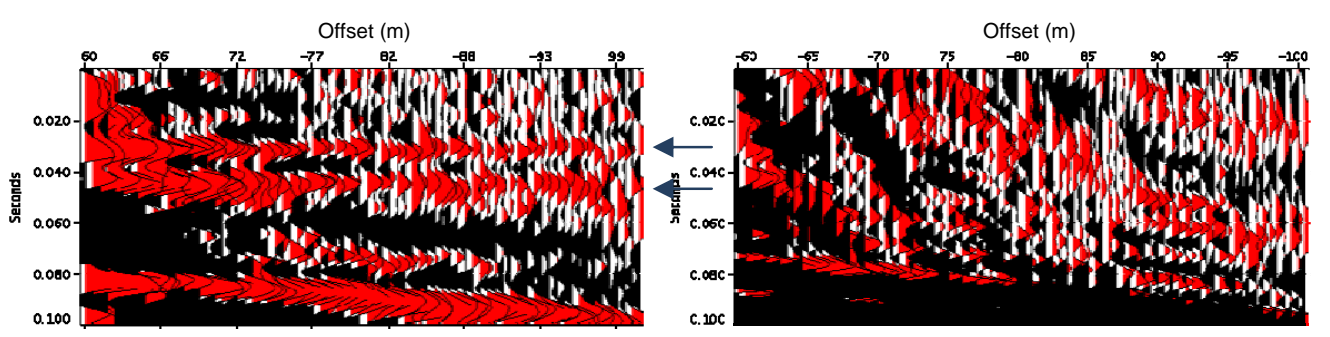

Figure 4: The top 100 ms of the vibroelectric (left) and seismic (right) data shown in Figure 3.

Figure 5 shows a combination of 19 shots from the walkaway test acquired with the 4-m dipole spacing sorted by offset for offsets of between 20 and $140 \mathrm{~m}$. The interfacial signal is clearly evident 


\section{Vienna ''11}

at $40 \mathrm{~ms}$ and extends from offsets of less than $40 \mathrm{~m}$ to offsets of more than $120 \mathrm{~m}$. The jitter between the traces is likely due to subtle variations in the depth of the water table (Figure 6).

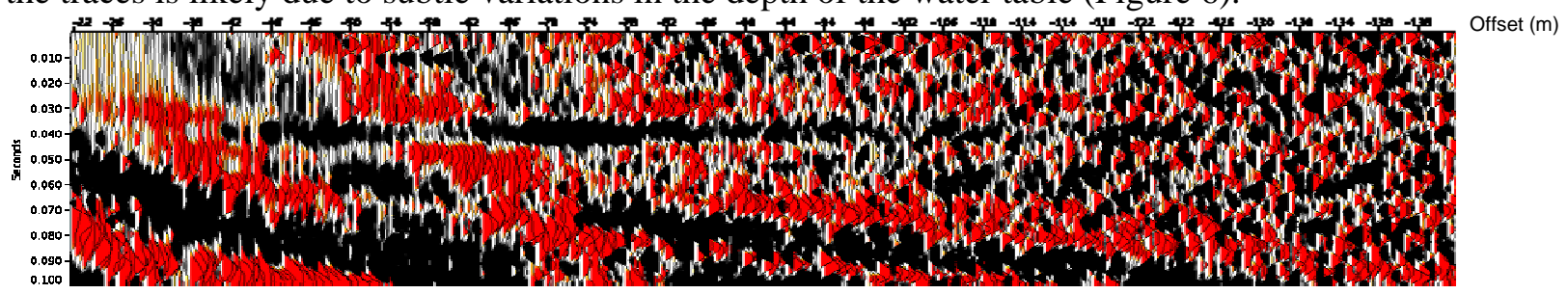

Figure 5: Supergather of 19 shots from the 4-m-dipole-spaced walkaway test.

\section{Conclusions}

The results of this experiment show that is it possible to map subsurface interfaces using the vibroelectric method. We successfully detected signals from shallow interfaces at offsets of up to $120-\mathrm{m}$, well within the spacing of most seismic surveys. Unlike previous seismoelectric surveys the stacking of multiple records was not required; the energy from a single sweep by the vibrator being sufficient. Unfortunately, due to the fast refractors in the area, it was not possible to confidently identify deeper signals although the application of more advanced processing procedures, or the acquisition or another test data set, may enable an idea of the

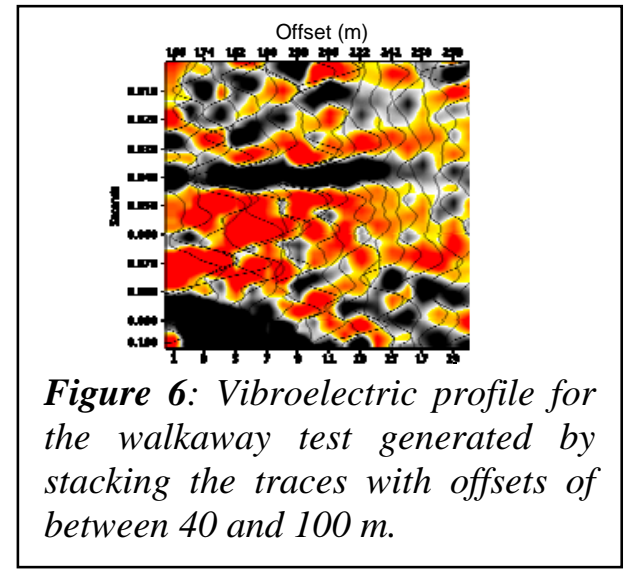
maximum depth of penetration to be established.

Given the need for a better understanding of the near surface and the advantages of integrated vibroelectric and vibroseis surveys detailed in the introduction, we believe that the vibroelectric method has the potential to become a useful tool in both understanding the near surface and improving seismic data quality. Because the source of the interfacial response is directly underneath the vibrator it is not necessary to record a significant range of offsets and thus the number of active channels recorded during a survey would be minimal (the profile shown in Figure 6 was generated using an average of just six traces).

\section{Acknowledgments}

Bruce Clulow, Dave Holmes, Peter Vermeer, Robin Davies, and others at WesternGeco for their help in organising the test. Elmar Strobach and Andrew Greenwood from Curtin University and Graham Cahill for helping with the acquisition. Anton Kepic and Karl Butler for the loan of the amplifiers.

\section{References}

Butler, K., and Russell, R. [1993] Subtraction of powerline harmonics from geophysical records. Geophysics, 58, 898-903.

Colombo, D., and Keho, T. [2010] The non-seismic data and joint inversion strategy for the near surface solution in Saudi Arabia. 80 ${ }^{\text {th }}$ SEG Annual Meeting, Expanded Abstracts, 1934-1938.

Dupuis, J., Butler, K., and Kepic, A. [2007] Seismoelectric imaging of the vadose zone of a sand aquifer. Geophysics, 72, A81-A85.

Haartsen, M., and Pride, S. [1997] Electroseismic waves from point sources in layered media. Journal of Geophysical Research, 102, 24745-24769.

Haines, S. [2004] Seismoelectric imaging of shallow targets (PhD thesis). Stanford University.

Kepic, A., and Rosid, M. [2004] Enhancing the seismoelectric method via a virtual shot gather. $74^{\text {th }}$ SEG Annual Meeting, Expanded Abstracts.

Sallas, J. [2010] How do hydraulic vibrators work? A look inside the black box. Geophysical Prospecting, 58, 3-17. 